\title{
Inflammatory bowel disease and anxiety: links, risks, and challenges faced
}

This article was published in the following Dove Press journal:

Clinical and Experimental Gastroenterology

23 March 2015

Number of times this article has been viewed

\author{
Ayman S Bannaga' \\ Christian P Selinger ${ }^{2}$ \\ 'Department of Gastroenterology, \\ Doncaster Royal Infirmary, Doncaster, \\ UK; ${ }^{2}$ Department of Gastroenterology, \\ St James University Hospital, Leeds, \\ UK
}

\begin{abstract}
Inflammatory bowel disease (IBD) causes severe physical symptoms and is also associated with psychological comorbidities. Abnormal anxiety levels are found in up to $40 \%$ of patients with IBD. Anxiety symptoms are often related to flares of IBD but may persist in times of remission. Detection of anxiety disorder (AD) in patients with IBD can be challenging. Patients with anxiety may also exhibit symptoms in keeping with functional gastrointestinal disorders (FGID). Evidence for the effectiveness of pharmacological and psychological therapies for anxiety stems from patients without IBD. Studies in patients with IBD have either been small or shown negative results. In light of this, a combined approach involving IBD physicians to improve disease control and psychologists or psychiatrists to treat anxiety is advised. This review examines the evidence of anxiety issues in IBD with a focus on extent of the problem, risk factors for anxiety, and the effectiveness of interventions.
\end{abstract}

Keywords: inflammatory bowel disease, Crohn's disease, ulcerative colitis, anxiety

\section{Introduction}

Inflammatory bowel disease (IBD) comprises two major gastrointestinal disorders namely Crohn's disease (CD) and ulcerative colitis (UC). It has a profound impact on a patient's life. IBD can affect people of all ages, but is most often diagnosed at a young age. It has a chronic nature and follows an unpredictable course with flares and remissions. Approximately $25 \%-50 \%$ of patients relapse annually. The etiology of IBD is not well recognized. It is now understood that it may relate to the complex interaction between genetic and environmental factors in vulnerable individuals. This interaction results in an inappropriate and exaggerated intestinal inflammatory response. ${ }^{1-3}$ IBD is a common condition. In the UK, CD affects around one in every 650 people, while UC affects around one in every 420 people. ${ }^{4}$ The incidence of CD in Europe ranges from 0.5-10.6 cases per 100,000 person-years, while the estimates for UC range from 0.9-24.3 per 100,000 person-years. ${ }^{5}$ There are great variations between countries, with generally higher incidences in more northern countries, and an increasing incidence has been noted in developing countries. Psychological effects from IBD and IBD medications are experienced by many patients with IBD. Anxiety, especially, can have a profound effect on quality of life, including the ability to work and have a family life. This paper reviews the current evidence to help clinicians looking after patients with IBD who experience anxiety.

\section{IBD symptoms, complications, and mortality}

The symptoms of IBD include abdominal pain, bloody diarrhea, and fatigue. Other bowel related symptoms are frequency of bowel motions, urgency to open the bowels, 
and occasionally, fecal incontinence. These symptoms cause immediate disruption of activities, but also have ongoing impacts on daily activities, including dietary restrictions, lifestyle changes, and maintaining close proximity to a toilet. More distal impacts include interference with work, school, parenting, social and leisure activities, relationships, and psychological well-being. ${ }^{6-8}$

IBD can have numerous complications involving all the systems of the body. CD can affect any part of the digestive tract from mouth to anus but most often leads to inflammation in the ileocaecal region. The main intestinal complications of CD include acute inflammation of the intestines, ulcers, obstruction, and perforation. Despite the use of immunosuppressants, around $80 \%$ of patients with CD require surgery in the long-term, most commonly for ileal stricturing or disease refractory to medical interventions. Patients with $\mathrm{CD}$ often require a second operation within a few years of the first intestinal resection. ${ }^{9}$ In contrast to UC, patients with $\mathrm{CD}$ can experience fistulating disease where abnormal connections between the bowel and other organs, or the skin, occur. This most commonly affects the perianal area. In contrast, UC only affects the colon in a continuous fashion extending proximally from the rectum. UC causes inflammation but does not lead to stricture formation or fistulation, usually. Surgery for UC can occur for a number of reasons. First, patients with acute severe colitis often require surgery. It is now clear that patients who after 3 days of intravenous steroid therapy and still have marked diarrhea ( $>8$ stools/24 hours) and with a CRP of more than $45 \mathrm{mg} / \mathrm{L}$, have a very high chance $(85 \%)$ of requiring urgent surgery. Approximately around 15\%-25\% of patients with a severe attack will require urgent colectomy, regardless of medical therapy given. ${ }^{10,11}$ Second, an elective colectomy is required for patients developing colorectal dysplasia or cancer, furthermore patients not responding to medical long term management also require colectomy. Up to $25 \%$ of patients with UC will ultimately require surgery. ${ }^{9}$

Surgical therapy often leads to the formation of a permanent ostomy which can be associated with body image disturbances. Problems arising from surgery include healing of the perineal wound, adhesion obstruction, and ileostomy dysfunction. Rarely, sexual dysfunction can also occur in males if a perimuscular excision of the rectum is made. ${ }^{10,11}$

Other extraintestinal manifestations occurring in CD patients include involvement of the skin, joints, and eyes. Women with CD have higher rates of miscarriage, particularly those with active disease during pregnancy. ${ }^{11}$ IBD patients are more prone to developing malignant disease, particularly who have UC, have increased risk for developing colorectal cancer when compared with the normal population. ${ }^{12}$ UC increases the risk of colorectal cancer to 2.4-fold, especially in male patients diagnosed at a young age and patients who have extensive colitis. ${ }^{13} \mathrm{UC}$ is also associated with primary sclerosing cholangitis, which puts UC patients at possible increased risk for malignant transformation to cholangiocarcinoma. ${ }^{14}$ IBD rarely results in mortality directly. There is largely normal mortality with UC and mild increase of mortality in CD. In UC, mortality rates are similar to those of the general population in most studies. On the other hand the majority of studies of CD mortality demonstrated 1.5-fold increases in the standardized mortality ratios, especially for those diagnosed at younger ages and requiring extensive or multiple resection surgery. ${ }^{15,16}$

As outlined, IBD can have a very severe physical impact on patients' health and their quality of life. Even at times of remission real concerns over the future course of disease remain, which can negatively affect the patients' personal outlook on life.

\section{Links between IBD and anxiety}

Anxiety disorder (AD) is defined as a feeling of unease, worry, and/or fear that patients are unable to control. It can be mild or severe, and last for a period of at least 6 months. ${ }^{17}$ Symptoms of AD have been outlined in Table 1.

IBD is a chronic disease, and it is well known that patients with chronic diseases have higher rates of anxiety compared to the general population. ${ }^{18-20} \mathrm{AD}$ occurs in IBD patients more often than expected by chance, and in particular with CD patients. ${ }^{21-26}$ UC patients also suffer from $A D$ at a higher rate than either the general population or groups of patients with other types of chronic diseases. ${ }^{26-28}$

A small study of 51 IBD patients in remission and 28 community controls reported a higher lifetime prevalence of $\mathrm{AD}$ in $\mathrm{CD}$ patients, compared to the control group. ${ }^{24}$ Another study that matched 79 IBD clinic patients with 36 healthy controls and used validated symptom patient report measures to assess anxiety symptoms found that IBD patients had higher anxiety levels than the control group. ${ }^{26}$ The prevalence of AD in IBD patients is increasing. ${ }^{28,29}$ In IBD patients, the rate of anxiety has been estimated between $29 \%$ and $35 \%$ during periods of remission and as high as $80 \%$ during relapses. ${ }^{30}$ Indeed the correlation between symptoms and anxiety levels seems strong. A Dutch study on 231 IBD patients suggested that up to $43 \%$ had high levels of anxiety, indicative of a psychiatric disorder and that anxiety symptoms and psychiatric complaints in IBD patients are undertreated. ${ }^{31}$ 
Table I Symptoms experienced in anxiety disorder

\begin{tabular}{l} 
General symptoms \\
Hot flushes or cold chills \\
Numbness or tingling sensations \\
Autonomic arousal symptoms \\
Palpitations \\
Sweating \\
Trembling or shaking \\
Symptoms concerning chest and abdomen \\
Difficulty breathing \\
Feeling of choking \\
Chest pain or discomfort \\
Nausea or abdominal distress \\
Symptoms concerning brain and mind \\
Feeling dizzy and unsteady \\
Faint or light-headed \\
Feelings that objects are unreal (derealization), or that one's self is \\
distant or "not really here" \\
Fear of losing control, going crazy, or passing out \\
Fear of dying \\
Symptoms of tension \\
Muscle tension or aches and pains \\
Restlessness and inability to relax \\
Feeling keyed up, or on edge, or of mental tension \\
A sensation of a lump in the throat, or difficulty with swallowing \\
Other nonspecific symptoms \\
Exaggerated response to minor surprises or being startled \\
Difficulty in concentrating, or mind going blank, because of worrying or \\
anxiety \\
Persistent irritability \\
Difficulty getting to sleep because of worrying \\
\hline
\end{tabular}

$\mathrm{AD}$ as a risk factor for IBD onset was also studied. One population based study found increased levels of AD among IBD patients, and it also found that AD may occur well before the onset of IBD, suggesting AD as a possible risk factor for developing IBD. ${ }^{32}$ Despite this study and others from the literature, there is no conclusive evidence that $\mathrm{AD}$ is a contributing risk for the onset of IBD onset. Whether AD truly predisposes patients to develop IBD remains controversial.

The symptoms of IBD can certainly lead to psychological changes among patients. Many studies suggested that mood disorders and psychological stress can trigger relapses in IBD. ${ }^{25,33-35}$ The etiology for these psychological changes remains unknown and is being studied by experimentation in animal models. In a study conducted on rats, researchers induced UC like inflammation using dextran sodium sulfate (DSS), they found that UC like inflammation in rats causes anxiety like behaviors, as well as ongoing abdominal discomfort. ${ }^{36}$ This model experiment goes some way in confirming the impression of many clinicians that physical symptoms can induce anxiety. Whether anxiety in patients with existing IBD can trigger a flare is less well understood. Mechanisms by which the stress through the nervous system can affect immune function at both systemic and gut mucosal levels are under research.

IBD patients commonly have overlap of symptoms suggestive of functional gastrointestinal disorders (FGID), including irritable bowel syndrome (IBS). This is not surprising as up to $30 \%$ of the general population display symptoms of IBS during their lifetime. In an Australian cohort, $66 \%$ of patients with IBD also met criteria for at least one FGID. The incidence of FGID was significantly higher in patients with higher anxiety levels. ${ }^{37}$ Anxiety can potentially exacerbate these disorders leading to greater psychological comorbidity and poorer quality of life. ${ }^{38,39}$ While increased symptoms are present in patients with AD it is unclear whether these symptoms are due to increased inflammation or overlapping FGID, as IBD symptoms scoring system cannot differentiate the origin of symptoms. Since it can be difficult to differentiate symptoms from FGID and IBD, physicians should, in the first instance, look for objective signs of inflammations. Any inflammation should be treated to induce remission from IBD, which may also improve anxiety and functional symptoms. If symptoms persist in patients with no objective evidence of active IBD, treatment for functional symptoms should be considered.

\section{Risk factors for developing anxiety in IBD}

Many risk factors associated with the development of anxiety in IBD patients have been described in the literature. These factors have been outlined in Table 2 .

Older age has been postulated as a risk factor; being 40 years or older was considered an independent predictor of impaired quality of life in a Korean study conducted on inactive IBD patients who have FGID and mood disorders. ${ }^{40}$ Female sex as risk factor for psychiatric disorders in IBD is controversial. The decrease in quality of life was significantly related with female sex. ${ }^{5}$ Female patients are more prone to develop depression. ${ }^{41,42}$ However, another study did not find a female predominance among anxious patients. ${ }^{30}$

IBD disease activity is closely linked to psychological symptoms as anxiety has also been observed more during

Table 2 Possible risk factor for AD in IBD

Psychological stress
Increasing age
Severe and active disease
Surgery and stoma
Patient education
Poor socioeconomic status

Abbreviations: $A D$, anxiety disorder; IBD, inflammatory bowel disease. 
flares of IBD patients. ${ }^{30,43}$ A study on UC patients concluded that endoscopically confirmed active mucosal inflammation is associated with more psychological stress. ${ }^{44}$ As such, the study demonstrated the importance of inflammation control for both the physical and psychological well-being of IBD patients (Figure 1). In another large cohort of IBD patients, risk factors for anxiety were severe and active disease. ${ }^{30}$

Many IBD patients require surgery and may end up with permanent stoma following removal of the colon, which is a huge lifelong change for them. In a small study on patients with CD and a stoma, it was found that patients had high rates of psychological comorbidity and low quality of life, and most of the patients were not receiving psychological help. It also concluded that psychological care is indicated for many of these patients. ${ }^{45}$ In another study IBD related surgery or hospitalization was associated with a significant risk for anxiety. ${ }^{46}$ These effects can be attributed to the severity of disease which necessitated hospital admission in the first instance, the side effects of any treatment received (especially surgery), and the hospital environment which is often seen as intimidating by patients.

Whether disease related patient education has an effect on the risk of developing anxiety is unclear. An Australian study found a correlation between patient knowledge and anxiety levels, suggesting that patients with better knowledge also had higher levels of anxiety. ${ }^{47}$ Whether anxious patients are more likely to search for information on IBD or whether better education creates anxiety remains unclear. A small Scandinavian study on group based patient education found that in 49 participants knowledge improved without an increase in anxiety scores. ${ }^{48}$ Further work is needed to evaluate the association between anxiety and knowledge and to develop targeted interventions that will improve knowledge and simultaneously reduce anxiety.

Perceived rather than actual stress may also play a role in the development of AD. Another study found that in UC, anxiety scores were associated with perceived stress and a

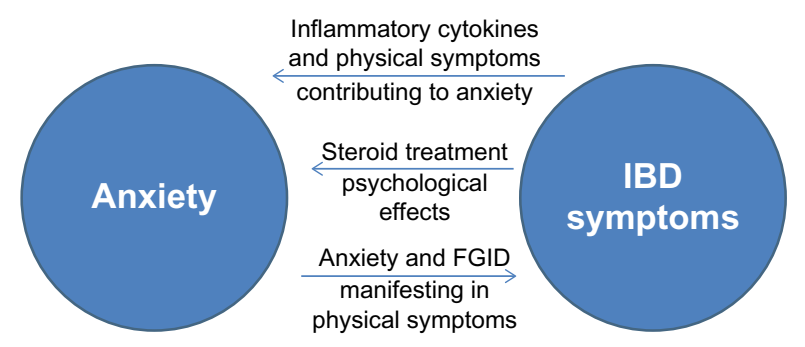

Figure I Interaction between anxiety and IBD symptoms.

Abbreviations: FGID, functional gastrointestinal disorders; IBD, inflammatory bowel disease. new diagnosis of IBD, while in $\mathrm{CD}$ patients anxiety was associated with perceived stress, abdominal pain, and lower socioeconomic status. ${ }^{30}$

Coping mechanisms play an important role as risk factors for flares in CD. ${ }^{34}$ Patients who engaged less in avoidance coping (avoiding to deal with stressors) were less likely to experience flares. ${ }^{34}$

The pathophysiology of the gut-brain axis may play an important role in the course of IBD, for both inflammation and psychological symptoms. TNF-alpha, a proinflammatory cytokine, has been postulated as a key factor. Further research in this field may reveal therapeutic targets in the future for both IBD and psychological symptom improvement. ${ }^{49}$

\section{Current challenges}

Given the association between IBD and AD, clinicians should ensure that detection and treatment of anxiety should be integral parts of IBD clinical care. Detecting AD should be a complement between the primary and secondary care clinicians looking after IBD patients, especially in IBD patients who have associated risks factors for developing AD.

Most IBD physicians have, however, little formal training in the detection and management of psychological comorbidities. This lack of training combined with the hesitancy of many patients to report psychological symptoms can delay the diagnosis and treatment of IBD related AD.

Formal screening questionnaires could overcome this issue. Such screening could occur during follow-up clinics, whether in the community or in specialized IBD clinics, with a structured interview about the symptoms of anxiety. There are screening tools clinicians can use to detect $\mathrm{AD}$, an example of a screening tool is the five item Anxiety and depression detector (Table 3 ). ${ }^{50}$ This detector uses five questions with yes or no answers. It was found to be sensitive, and had reasonable specificity for detecting AD across different sex and racial groups. Another screening tool is the Luebeck Interview for Psychosocial Screening in Patients

Table 3 Anxiety and depression detector

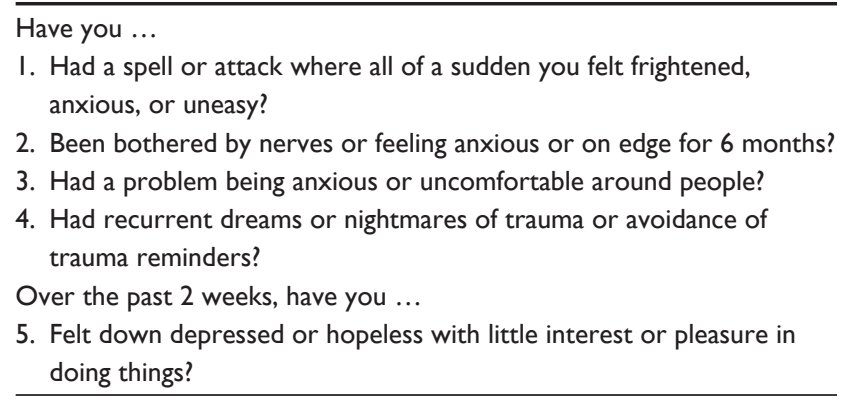

I. Had a spell or attack where all of a sudden you felt frightened, anxious, or uneasy?

2. Been bothered by nerves or feeling anxious or on edge for 6 months?

3. Had a problem being anxious or uncomfortable around people?

4. Had recurrent dreams or nightmares of trauma or avoidance of trauma reminders?

you ... doing things? 
with IBD. ${ }^{51}$ This scale provides a broader scope of information for patient management. It covers areas including social support, depression and anxiety caused by IBD, and takes an average of 5-10 minutes to administer. Awareness of these screening tools may help in the recognition of patients with anxiety to consider them for appropriate therapy. However preclinic questionnaires that ask for all desirable information (physical symptoms, psychological symptoms, quality of life, medication adherence, etc) can easily become very lengthy and burdensome to the patient. As such a difficult balance between desirable screening and practical approaches has to be struck.

Many IBD patients with AD are under treated and this leads to disability and some even have a stigma against seeking treatment. ${ }^{52-54}$ It has been shown that therapy directed toward psychological comorbidity might independently improve the quality of life in IBD patients. ${ }^{38}$ Treatment for AD should be considered once patients with risk factors screen positive for $\mathrm{AD}$, and the patient is showing features of poor health related quality of life (HRQoL). ${ }^{55}$ Management should ideally involve a multidisciplinary team of specialists, including a gastroenterologist and a psychologist or psychiatrist, as treatment for AD involves pharmacological and psychological aspects. A multidisciplinary approach is required to ensure optimization of medical IBD therapy as well as therapy for AD to achieve full control of IBD and AD together.

In general, management of otherwise healthy patients with $\mathrm{AD}$ includes psychological therapies and the use of pharmacological agents. Selective serotonin reuptake inhibitors (SSRIs) are generally used as first-line agents, followed remotely by tricyclic antidepressants (TCAs), and serotonin norepinephrine reuptake inhibitors (SNRIs). These agents have been well established in the literature for being effective in the treatment of AD. ${ }^{56}$ The other aspect in $\mathrm{AD}$ management is the psychological treatment including cognitive behavioral therapy (CBT). The effectiveness of CBT in treating AD has been demonstrated by a number of large scale studies and meta-analyses. ${ }^{57-59}$ Indeed, a combination of psychological treatment and pharmacotherapy seems to show the greatest treatment benefits. ${ }^{57}$

There are less data on the effectiveness for interventions treating $\mathrm{AD}$ in IBD patients. A small single-arm study of nine patients has found that $\mathrm{AD}$ symptoms can be improved by CBT in adolescents with IBD. A decrease in anxiety, pain, and disease severity was also observed. ${ }^{60}$ A Swedish study examined the effects of a group-based education program for 49 patients with IBD and high anxiety levels. ${ }^{48}$
The intervention failed to improve anxiety levels and IBD symptoms, but led to improved patient knowledge.

There has been a multitude of studies addressing the impact of psychological interventions (psychotherapy, patient education, and relaxation techniques) in IBD in general, but these studies have not specifically looked at IBD patients with confirmed AD. A recent Cochrane review summarized the evidence for general adult IBD patients and found no effect of psychotherapy on quality of life, depressive symptoms, or clinical remission from IBD. ${ }^{61}$ While there is conclusive evidence for the effectiveness of psychological therapies and the use of pharmacological agents for patients with $\mathrm{AD}$, there is a lack of evidence for an improvement in anxiety in patients with IBD.

Another treatment modality that has not been fully evaluated yet is gut-directed hypnotherapy (GHT). GHT has been used successfully in FGID. A small case series of 15 patients with IBD responded well to hypnotherapy. Improvements in bowel symptoms and quality of life were noted, but this may relate to improving function symptoms rather than inflammation. ${ }^{62}$ Further evidence from hypnotherapy stems from a clinical trial of 54 patients with UC in clinical remission. Patients randomized to hypnotherapy were less likely to experience a flare during 1 year follow-up, compared to controls. ${ }^{63}$

\section{Conclusion and expert opinion}

Psychological symptoms are very common in patients with IBD and can lead to reduced quality of life. AD can lead to IBD related symptoms and additional AD related physical symptoms. At least $40 \%$ of patients with IBD will exhibit abnormal anxiety levels. This may often go undetected as physician awareness and training in this aspect is suboptimal. Detection of $\mathrm{AD}$ requires an active approach and a good patient-doctor relationship. Active IBD can often cause anxiety, and induction of clinical remission may improve AD symptoms too.

The evidence for interventions is limited to AD in general, as studies in patients with IBD showed either negative results or were too small to deliver meaningful results. Studies of psychological interventions in patients with IBD have not produced convincing results, but in the subgroup of patients with anxiety this may be different. A multidisciplinary approach is advised, with the IBD physician aiming to induce clinical remission and a psychologist to provide input to coordinate psychological and pharmacological interventions for AD (Table 4).

There is a need for further research into the effectiveness of interventions aimed at improving $\mathrm{AD}$ in patients with IBD. 


\section{Table 4 Conclusion}

Be aware of $A D$ in IBD patients

Screen for $A D$ in patients with possible risk factors

Aim for full control of IBD

Initiate the treatment in multidisciplinary fashion

Involve patients in the choice of psychological therapy

Abbreviations: AD, anxiety disorder; IBD, inflammatory bowel disease.

It would also be desirable for patients and mental health specialists to participate in future guideline development on psychosocial issues in IBD.

\section{Disclosure}

Dr Selinger has received research grants from Shire, Nycomed, Ferring, Warner Chilcott, and Abbvie. He has provided consultancy to Abbvie, Dr Falk, and Takeda. He has received speaking honoraria from MSD, Ferring, and Abbvie. The authors report no other conflicts of interest.

\section{References}

1. Podolsky D. Inflammatory bowel disease. $N$ Engl J Med. 2002;347: 417-429.

2. Kucharzik T, Maaser C, Lugering A, et al. Recent understanding of IBD pathogenesis: implications for future therapies. Inflamm Bowel Dis. 2006;12:1068-1083.

3. Sartor R. Mechanisms of disease: pathogenesis of Crohn's disease and ulcerative colitis. Nat Clin Pract Gastroenterol Hepatol. 2006;3: 390-407.

4. The Publications Team. Information about IBD. Available from: http:// www.crohnsandcolitis.org.uk/information-and-support/informationabout-ibd/what-is-IBD. Accessed December 6, 2014.

5. Magalhães J, Castro F, Carvalho P, et al. Quality of life in patients with inflammatory bowel disease: importance of clinical, demographic and psychosocial factors. Arq Gastroenterol. 2014;51:192-197.

6. Devlen J, Beusterien K, Yen L, et al. The burden of inflammatory bowel disease: a patient-reported qualitative analysis and development of a conceptual model. Inflamm Bowel Dis. 2014;20:545-552.

7. Papathanasopoulos A, Van Oudenhove L, Katsanos K, et al. Severity of fecal urgency and incontinence in inflammatory bowel disease: clinical, manometric and sonographic predictors. Inflamm Bowel Dis. 2013;19:2450-2456.

8. Dibley L, Norton C. Experiences of fecal incontinence in people with inflammatory bowel disease: self-reported experiences among a community sample. Inflamm Bowel Dis. 2013;19:1450-1462.

9. Selinger C, Andrews J, Titman A, et al. Long-term follow-up reveals low incidence of colorectal cancer, but frequent need for resection, among Australian patients with inflammatory bowel disease. Clin Gastroenterol Hepatol. 2014;12:644-650.

10. Jewell D. Ulcerative Colitis. In: Warrell D, Cox T, Firth J, editors. Oxford Textbook of Medicine. 5th ed. Oxford, UK: Oxford University Press; 2010:2371-2383.

11. Parkes M. Crohn's Disease. In: Warrell D, Cox T, Firth J editors. Oxford Textbook of Medicine. 5th ed. Oxford, UK: Oxford University Press; 2010:2361-2370.

12. Lutgens $M$, Vermeire $S$, Van Oijen $M$, et al. A rule for determining risk of colorectal cancer in patients with inflammatory bowel disease. Clin Gastroenterol Hepatol. 2015;13:148-154.

13. Scarpa M, Castagliuolo I, Castoro C, et al. Inflammatory colonic carcinogenesis: a review on pathogenesis and immunosurveillance mechanisms in ulcerative colitis. World J Gastroenterol. 2014;20:6774-6785.
14. Tyson G, El-Serag H. Risk factors of cholangiocarcinoma. Hepatology. 2011;54:173-184.

15. Selinger C, Leong R. Mortality from inflammatory bowel diseases. Inflamm Bowel Dis. 2012;18:1566-1572.

16. Monstada I, Øistein H, Solberga I, et al. Clinical course and prognosis in ulcerative colitis: results from population-based and observational studies. Ann Gastroenterol. 2014;27:95-104.

17. World Health Organization. The ICD-10 Classification of Mental and Behavioural Disorders. Available from: http:/www.who.int/ classifications/icd/en/bluebook.pdf. Accessed December 6, 2014.

18. Katon W, Ciechanowski P. Impact of major depression on chronic medical illness. J Psychosom Res. 2002;53:859-863.

19. Patten S, Beck C, Kassam A, et al. Long-term medical conditions and major depression: strength of association for specific conditions in the general population. Can J Psychiatry. 2005;50:195-202.

20. Scott K, Bruffaerts R, Tsang A, et al. Depression-anxiety relationships with chronic physical conditions: results from the World Mental Health surveys. J Affect Disord. 2007;103:113-120.

21. Andrews H, Barczak P, Allan R. Psychiatric illness in patients with inflammatory bowel disease. Gut. 1987;28:1600-1604.

22. Helzer J, Chammas S, Norland C, et al. A study of the association between Crohn's disease and psychiatric illness. Gastroenterology. 1984;86:324-330.

23. Schwartz R, Schwartz I. Psychiatric disorders associated with Crohn's disease. Int J Psychiatry Med.1982;12:67-73.

24. Tartar R, Switala J, Carra J, et al. Inflammatory bowel disease: psychiatric status of patients before and after disease onset. Int J Psychiatry Med. 1987; 17:173-181.

25. Walker E, Roy-Byrne P, Katon W, et al. Psychiatric illness and irritable bowel syndrome: a comparison with inflammatory bowel disease. Am J Psychiatry.1990;147:1656-1661.

26. Addolorato G, Capristo E, Stefanini G, et al. Inflammatory bowel disease: a study of the association between anxiety and depression, physical morbidity, and nutritional status. Scand J Gastroenterol. 1997;32:1013-1021.

27. Magni G, Bernasconi G, Mauro P, et al. Psychiatric diagnoses in ulcerative colitis. A controlled study. Br J Psychiatry.1991;158:413-415.

28. Robertson D, Ray J, Diamond I, et al. Personality profile and affective state of patients with inflammatory bowel disease. Gut. 1989;30: 623-626.

29. Goodhand J, Wahed M, Mawdsley J, et al. Mood disorders in inflammatory bowel disease: relation to diagnosis, disease activity, perceived stress, and other factors. Inflamm Bowel Dis. 2012;18:2301-2309.

30. Nahon S, Lahmek P, Durance $C$, et al. Risk factors of anxiety and depression in inflammatory bowel disease. Inflamm Bowel Dis. 2012;18: 2086-2091.

31. Bennebroek Evertsz F, Thijssens N, Stokkers P. et al. Do inflammatory bowel disease patients with anxiety and depressive symptoms receive the care they need? J Crohns Colitis. 2012;6:68-76.

32. Walker J, Ediger J, Graff L, et al. The Manitoba IBD cohort study: a population-based study of the prevalence of lifetime and 12-month anxiety and mood disorders. Am J Gastroenterol. 2008;103:1989-1997.

33. Levenstein S, Prantera C, Varvo V, et al. Stress and exacerbation in ulcerative colitis: a prospective study of patients enrolled in remission. Am J Gastroenterol. 2000;95:1213-1220.

34. Bitton A, Dobkin P, Edwardes M, et al. Predicting relapse in Crohn's disease: a biopsychosocial model. Gut. 2008;57:1386-1392.

35. Mawdsley J, Rampton D. Psychological stress in inflammatory bowel disease. Gut. 2005;54:1481-1491.

36. Chen J, Winston J, Fu Y, et al. Genesis of anxiety, depression and ongoing abdominal discomfort in ulcerative colitis-like colon inflammation. Am J Physiol Regul Integr Comp Physiol. 2015;1:18-27.

37. Filipovic B, Filipovic B. Psychiatric comorbidity in the treatment of patients with inflammatory bowel disease. World $J$ Gastroenterol. 2014;20:3552-3563.

38. Bryant R, van Langenberg D, Holtmann G, et al. Functional gastrointestinal disorders in IBD. J Gastroenterol Hepatol. 2011;26:916-923. 
39. Kovacs Z, Kovacs F. Depressive and anxiety symptoms, dysfunctional attitudes, and social aspects in irritable bowel syndrome and inflammatory bowel disease. Int J Psych Med. 2007;37:245-255.

40. Kim E, Cho K, Park K, et al. Predictive factors of impaired quality of life in Korean patients with inactive inflammatory bowel disease: association with functional gastrointestinal disorders and mood disorders. J Clin Gastroenterol. 2013;47:38-44.

41. Trachter A, Rogers A, Leiblum S. Inflammatory bowel disease in women: impact on relationship and sexual health. Inflamm Bowel Dis. 2002;8:413-421.

42. Fuller-Thomson E, Sulman J. Depression and inflammatory bowel disease: findings from two nationally representative Canadian surveys. Inflamm Bowel Dis. 2006;12:697-707.

43. Levenstein S, Prantera C, Varvo V, et al. Psychological stress and disease activity in ulcerative colitis: a multidimensional cross-sectional study. Am J Gastroenterol. 1994;89:1219-1225.

44. Bernstein C, Singh S, Graff L, et al. A prospective population based study of triggers of symptomatic flares in IBD. Am J Gastroenterol. 2010;105:1994-2002.

45. Knowles S, Wilson J, Wilkinson A, et al. Psychological well-being and quality of life in Crohn's disease patients with an ostomy: a preliminary investigation. J Wound Ostomy Continence Nurs. 2013;40:623-629.

46. Ananthakrishnan A, Gainer V, Cai T, et al. Similar risk of depression and anxiety following surgery or hospitalization for Crohn's disease and ulcerative colitis. Am J Gastroenterol. 2013;108:594-601.

47. Selinger C, Lal S, Eaden J, et al. Better disease specific patient knowledge is associated with greater anxiety in inflammatory bowel disease. J Crohns Colitis. 2013;7:214-218.

48. Larsson K, Sundberg Hjelm M, Karlbom U, et al. A group-based patient education programme for high-anxiety patients with Crohn disease or ulcerative colitis. Scand J Gastroenterol. 2003;38:763-769.

49. Bonaz B and Bemestein C. Brain-gut interactions in inflammatory bowel disease. Gastroenterology. 2013;144:36-49.

50. Means-Christensen A, Sherbourne C, Roy-Byrne P, et al. Using five questions to screen for five common mental disorders in primary care: diagnostic accuracy of the Anxiety and Depression Detector. Gen Hosp Psychiatry. 2006;28:108-118.

51. Kunzendorf S, Jantschek G, Straubinger K, et al. The Luebeck interview for psychosocial screening in patients with inflammatory bowel disease. Inflamm Bowel Dis. 2007;13:33-41.
52. Collins K, Westra H, Dozois D, et al. Gaps in accessing treatment for anxiety and depression: challenges for the delivery of care. Clin Psychol Rev. 2004;24:583-616.

53. Wang P, Lane M, Olfson M, et al. Twelve-month use of mental health services in the United States: results from the national co-morbidity survey replication. Arch Gen Psychiatry. 2005;62:629-640.

54. Prins M, Verhaak P, Bensing J, et al. Health beliefs and perceived need for mental health care of anxiety and depression-the patients' perspective explored. Clin Psychol Rev. 2008;28:1038-1058.

55. Iglesias-Rey M, Barreiro-de Acosta M, Caamaño-Isorna F, et al Psychological factors are associated with changes in the health-related quality of life in inflammatory bowel disease. Inflamm Bowel Dis. 2014;20:92-102.

56. Hansen R, Gartlehner G, Lohr K, et al. Efficacy and safety of secondgeneration antidepressants in the treatment of major depressive disorder. Ann of Intern Med. 2005;143:415-426.

57. Bandelow B, Seidler-Brandler U, Becker A, et al. Meta-analysis of randomized controlled comparisons of psychopharmacological and psychological treatments for anxiety disorders. World J Biol Psychiatry. 2007;8:175-187.

58. Norton P, Price E. A meta-analytic review of adult cognitive-behavioral treatment outcome across the anxiety disorders. J Nerv Ment Dis. 2007;195:521-531.

59. Cuijpers P, van Straten A, van Oppen P, et al. Are psychological and pharmacologic interventions equally effective in the treatment of adult depressive disorders? A meta-analysis of comparative studies. J Clin Psychiatry. 2008;69:1675-1685.

60. Reigada L, Benkov K, Bruzzese J, et al. Integrating illness concerns into cognitive behavioral therapy for children and adolescents with inflammatory bowel disease and co-occurring anxiety. J Spec Pediatr Nurs. 2013;18:133-143.

61. Timmer A, Preiss J, Motschall E, et al. Psychological interventions for treatment of inflammatory bowel disease. Cochrane Database Syst Rev. 2011;2:CD006913.

62. Moser G. The role of hypnotherapy for the treatment of inflammatory bowel diseases. Expert Rev Gastroenterol Hepatol. 2014;8: 601-606.

63. Keefer L, Taft T, Kiebles J, et al. Gut-directed hypnotherapy significantly augments clinical remission in quiescent ulcerative colitis. Aliment Pharmacol Ther. 2013;38:761-771.
Clinical and Experimental Gastroenterology

\section{Publish your work in this journal}

Clinical and Experimental Gastroenterology is an international, peerreviewed, open access journal, publishing all aspects of gastroenterology in the clinic and laboratory, including: Pathology, pathophysiology of gastrointestinal disease; Investigation and treatment of gastointes tinal disease; Pharmacology of drugs used in the alimentary tract;

\section{Dovepress}

Immunology/genetics/genomics related to gastrointestinal disease. This journal is indexed on CAS. The manuscript management system is completely online and includes a very quick and fair peer-review system. Visit http://www.dovepress.com/testimonials.php to read real quotes from published authors. 